\title{
Beneficial effect of whole-grain wheat on liver fat: a role for the gut microbiota?
}

\section{Philippe Gérard}

Micalis Institute, Université Paris-Saclay, INRAE, AgroParisTech, Jouy-en-Josas, France

Correspondence to: Philippe Gérard, PhD. Micalis Institute, Université Paris-Saclay, INRAE, AgroParis'Tech, Building 442, 78350 Jouy-en-Josas, France. Email: philippe.gerard@inrae.fr.

Comment on: van Trijp MPH, Schutte S, Esser D, et al. Minor Changes in the Composition and Function of the Gut Microbiota During a 12-Week Whole Grain Wheat or Refined Wheat Intervention Correlate with Liver Fat in Overweight and Obese Adults. J Nutr 2021;151:491-502.

Submitted Aug 12, 2021. Accepted for publication Aug 26, 2021.

doi: 10.21037/hbsn-21-332

View this article at: https://dx.doi.org/10.21037/hbsn-21-332

In meta-analyses of prospective studies, high whole grain (WG) intake has been acknowledged for its potential role in lowering risk of type 2 diabetes, cancer, cardiovascular disease and all cause mortality $(1,2)$. Unlike Refined wheat (RW) flours from which the bran and germ of the wheat kernel have been removed, whole-grain wheat (WGW) products retain these elements and are rich sources not only of dietary fiber, but also of betaine, choline, important minerals and vitamins, components with antioxidant properties, and other phytochemicals (3). They are thus considered nutritionally superior but previous WG interventions in human subjects have reported conflicting results in improvements in health parameters.

In this context, The Graandioos study has been performed in the Netherlands to investigate the benefits of WGW. In this randomized, doubleblind, parallel trial, 50 overweight female and male middle-aged participants were first aligned on a 4-wk RW period. Next, participants were randomly assigned to a 12 -wk RW or WGW intervention. Plasma samples were taken after overnight fasting and postprandially and thirty-one biomarkers were quantified focusing on metabolism, liver, cardiovascular health, and inflammation. The results of the Graandioos study, described in two previous papers $(4,5)$, revealed that the WGW intervention promotes liver and inflammatory resilience in overweight subjects and prevents the increase in liver fat observed in RW intervention.

In the last decade, a contribution of the gut microbiota in NAFLD has been revealed $(6,7)$. Indeed, a dysbiosis is associated with NAFLD and this perturbed gut microbiota can result in increased gut permeability and facilitation of the passage of LPS and other inflammatory factors to the blood, decreased choline availability, changes in bile acid composition and increased ethanol production in the intestine. The dysbiosis also impacts energy harvest from the diet and alters the hepatic expression of genes involved in de novo lipogenesis. Altogether, these microbiota-associated mechanisms may influence steatosis development and fecal microbiota transplants recently confirmed that the gut bacteria affect hepatic lipid accretion (8).

Considering this influence of the gut bacteria on hepatic lipid metabolism, a role for the gut microbiota in the liver improvements effects of WGW intervention can be envisaged. To test this hypothesis, In the recently published paper (9) the authors analyzed the fecal microbiota composition and functionality in samples obtained from the Grandioos study, and correlated intervention-induced changes in gut bacteria with changes in liver health parameters. No significant clustering effect was found between baseline and postintervention within and between groups based on overall microbiota profiles indicating minor impact of the intervention on gut microbiota. This is not surprising considering the relatively modest differences in diet composition, only altering wheat products. Moreover both RW and WGW interventions did not change microbial diversity. Nevertheless, the WGW intervention increased 3 bacterial taxa (within the Ruminococcaceae family) and decreased 1 bacterial taxon (within the Lachnospiraceae family), whereas the 
RW intervention mostly decreased these bacteria. Maybe more importantly, WGW intervention showed a trend toward increased predicted fermentation pathways by the microbiota (in particular pathways for butyrate production), whereas RW intervention showed opposite effects. These findings could be explained by the decreased fiber content in the RW intervention products. Interestingly, it has been previously shown that butyrate can decrease intestinal inflammation and influence liver fat through stimulation of hepatic fat oxidation via activation of AMP-activated protein kinase (10). Based on these findings and on the previously published results showing that the 12 -wk RW intervention significantly increased liver fat, whereas WGW intervention did not, the authors hypothesized that WGW might improve steatosis indirectly via increased cereal fiber fermentation and SCFA production indicating a role for the gut microbiota in their beneficial effects. However, this hypothesis remains unproven due to several limitations of the study, including small sample size, prediction of microbial functionality from $16 \mathrm{~S}$ rRNA sequencing data instead of metagenomics, or absence of SCFA measurements in feces. Therefore, the potential health effects of replacement of RW by WGW and contribution of the gut microbiota in those effects still deserve further investigation.

\section{Acknowledgments}

Funding: None.

\section{Footnote}

Provenance and Peer Review: This article was commissioned by the editorial office of Hepatobiliary Surgery and Nutrition. The article did not undergo external peer review.

Conflicts of Interest: The author has completed the ICMJE uniform disclosure form (available at https://hbsn. amegroups.com/article/view/10.21037/hbsn-21-332/coif). The author has no conflicts of interest to declare.

Ethical Statement: The author is accountable for all aspects of the work in ensuring that questions related to the accuracy or integrity of any part of the work are appropriately investigated and resolved.

Open Access Statement: This is an Open Access article distributed in accordance with the Creative Commons
Attribution-NonCommercial-NoDerivs 4.0 International License (CC BY-NC-ND 4.0), which permits the noncommercial replication and distribution of the article with the strict proviso that no changes or edits are made and the original work is properly cited (including links to both the formal publication through the relevant DOI and the license). See: https://creativecommons.org/licenses/by-nc-nd/4.0/.

\section{References}

1. Aune D, Keum N, Giovannucci E, et al. Whole grain consumption and risk of cardiovascular disease, cancer, and all cause and cause specific mortality: systematic review and dose-response meta-analysis of prospective studies. BMJ 2016;353:i2716.

2. Chen GC, Tong X, Xu JY, et al. Whole-grain intake and total, cardiovascular, and cancer mortality: a systematic review and meta-analysis of prospective studies. Am J Clin Nutr 2016;104:164-72.

3. Shewry PR, Hey SJ. The contribution of wheat to human diet and health. Food Energy Secur 2015;4:178-202.

4. Schutte S, Esser D, Hoevenaars FPM, et al. A 12wk whole-grain wheat intervention protects against hepatic fat: the Graandioos study, a randomized trial in overweight subjects. Am J Clin Nutr 2018;108:1264-74.

5. Hoevenaars FPM, Esser D, Schutte S, et al. Whole Grain Wheat Consumption Affects Postprandial Inflammatory Response in a Randomized Controlled Trial in Overweight and Obese Adults with Mild Hypercholesterolemia in the Graandioos Study. J Nutr 2019;149:2133-44.

6. Fei N, Bruneau A, Zhang X, et al. Endotoxin Producers Overgrowing in Human Gut Microbiota as the Causative Agents for Nonalcoholic Fatty Liver Disease. mBio 2020;11:e03263-19.

7. Safari Z, Gérard P. The links between the gut microbiome and non-alcoholic fatty liver disease (NAFLD). Cell Mol Life Sci 2019;76:1541-58.

8. Burz SD, Monnoye M, Philippe C, et al. Fecal Microbiota Transplant from Human to Mice Gives Insights into the Role of the Gut Microbiota in NonAlcoholic Fatty Liver Disease (NAFLD). Microorganisms 2021;9:199.

9. van Trijp MPH, Schutte S, Esser D, et al. Minor Changes in the Composition and Function of the Gut Microbiota During a 12-Week Whole Grain 
Wheat or Refined Wheat Intervention Correlate with Liver Fat in Overweight and Obese Adults. J Nutr 2021;151:491-502.

Cite this article as: Gérard P. Beneficial effect of whole-grain wheat on liver fat: a role for the gut microbiota? HepatoBiliary Surg Nutr 2021;10(5):708-710. doi: 10.21037/hbsn-21-332
10. van der Hee B, Wells JM. Microbial Regulation of Host Physiology by Short-chain Fatty Acids. Trends Microbiol 2021;29:700-12. 